Research article

\title{
PKQuest: measurement of intestinal absorption and first pass metabolism - application to human ethanol pharmacokinetics David G Levitt
}

Address: Department of Physiology 6-125 Jackson Hall 321 Church St. S. E. Minneapolis, MN 55455, USA

E-mail: levitt@dcmir.med.umn.edu

Published: 15 August 2002

BMC Clinical Pharmacology 2002, 2:4
Received: 4 April 2002

Accepted: 15 August 2002

This article is available from: http://www.biomedcentral.com//472-6904/2/4

(C) 2002 Levitt; licensee BioMed Central Ltd. This article is published in Open Access: verbatim copying and redistribution of this article are permitted in all media for any non-commercial purpose, provided this notice is preserved along with the article's original URL.

\begin{abstract}
Background: PKQuest, a new physiologically based pharmacokinetic (PBPK) program, is applied to human ethanol data. The classical definition of first pass metabolism (FPM) based on the differences in the area under the curve (AUC) for identical intravenous and oral doses is invalid if the metabolism is non-linear (e.g. ethanol). Uncertainties in the measurement of FPM have led to controversy about the magnitude of gastric alcohol metabolism. PKQuest implements a new, rigorous definition of FPM based on finding the equivalent intravenous input function that would produce a blood time course identical to that observed for the oral intake. This input function equals the peripheral availability (PA) and the FPM is defined by: FPM = Total oral dose - PA. PKQuest also provides a quantitative measurement of the time course of intestinal absorption.

Methods: PKQuest was applied to previously published ethanol pharmacokinetic data.

Results: The rate of ethanol absorption is primarily limited by the rate of gastric emptying. For oral ethanol with a meal: absorption is slow ( $\approx 3$ hours) and the fractional PKQuest FPM was $36 \%$ $(0.15 \mathrm{gm} / \mathrm{Kg}$ dose $)$ and $7 \%(0.3 \mathrm{gm} / \mathrm{Kg})$. In contrast, fasting oral ethanol absorption is fast $(\approx 50$ minutes) and FPM is small.

Conclusions: The standard AUC and one compartment methods significantly overestimate the FPM. Gastric ethanol metabolism is not significant. Ingestion of a coincident meal with the ethanol can reduce the peak blood level by about 4 fold at low doses. PKQuest and all the examples are freely available on the web at [www.pkquest.com].
\end{abstract}

\section{Background}

The expression "first pass metabolism" (FPM) refers to the metabolism that an ingested compound undergoes in its passage through the gut and liver before reaching the systemic circulation. It is a useful concept for a drug because it provides information about the relative therapeutic effect of an orally administered drug relative to its intravenous administration (IV). The standard approach to quantitate FPM is to compare the area under the curve (AUC) for equal IV and oral doses. The fractional FPM is then defined as the fractional difference in these two areas:

(1) Fractional $\cdot$ First $\cdot$ Pass $\cdot$ Metabolism $=\frac{A U C(I V)-A U C(\text { oral })}{A U C(I V)}$

The mathematical basis of this definition is based on the following arguments: Given some time dependent input 
to the systemic compartment $(\mathrm{I}(\mathrm{t}))$, and some removal rate from the systemic compartment $(\mathrm{Q}(\mathrm{t}))$, the integral over all time of these two rates must be equal:

$$
\text { Total } \cdot \text { Input }=\int_{0}^{\infty} I(t) d t=\int_{0}^{\infty} Q(t) d t
$$

If the removal rate has a linear dependence on concentration $(\mathrm{Q}(\mathrm{t})=\mathrm{KC}(\mathrm{t}))$ then this total input can be related to the AUC:

$$
\text { Total } \cdot \text { Input }=\int_{0}^{\infty} Q(t) d t=K \int_{0}^{\infty} C(t) d t=K(\text { AUC })
$$

Since the total input to the systemic compartment is proportional to the AUC (eq. 3), the fractional difference in the AUC for an oral and IV dose (eq 1) is a measure of the amount of the oral drug that never reached the systemic compartment because of FPM.

This standard operational definition of FPM (eq. 1) is crucially dependent on the assumption that the removal rate is linear. If this assumption is invalid, then use of this definition can return very misleading results. A recent review has described the confusion that has resulted from applying this definition to human ethanol pharmacokinetics, a compound that has extremely non-linear liver metabolism [1].

For the non-linear case, the intuitive idea that FPM is equal to the fraction of the absorbed drug that is metabolized in its first pass through the liver is no longer valid [2]. Consider the case where GI absorption is occurring at a time when the systemic drug concentration is so high that the liver metabolism is completely saturated and the metabolic rate is constant, independent of concentration. Clearly, at this time, the rate of drug metabolism for an oral input must be identical to that for an IV input so that FPM must be zero, even though a large fraction of the absorbed drug may be metabolized in its first pass through the liver. This metabolized GI component is simply displacing systemic drug that would otherwise have been metabolized.

In order to avoid these problems, Lieber and colleagues [3] introduced a new definition of FPM, based on estimating the "Peripheral Availability" (PA) of ethanol. The PA is defined as the total amount of ethanol that reaches the systemic circulation. Gentry et al. [3] estimated the value of PA by assuming that the tail of the IV ethanol venous concentration curve can be approximated by a one compartment model in which the ethanol rate of metabolism (M) was defined by a Michaelis-Menten function $(\mathrm{M}=$ $\mathrm{VmC} /(\mathrm{Km}+\mathrm{C}))$. The values of $\mathrm{Vm}$ and $\mathrm{Km}$ are found by curve fitting, and then PA is defined as the integral of $\mathrm{M}$ from time 0 to a long time when no ethanol is present. Thus, PA is defined as the total amount of ethanol that was metabolized from the systemic circulation. The FPM is then defined as the difference between the PA for an IV infusion and the PA for the oral intake of an identical dose. This one compartment definition of FPM has become the standard definition in some laboratories [4].

This definition of FPM of Gentry et al. [3] is inaccurate because it is a poor approximation to treat ethanol metabolism as a one compartment system (see below). Levitt and Levitt [2] described an approach to estimate PA using a two compartment model. They distinguished the liver ethanol that came directly from the GI tract from the recirculating ethanol, and derived an accurate approximation for this recirculating component. This 2 compartment approach provides a significantly more accurate (and smaller) estimate of FPM than the one compartment model.

The 2 compartment model still makes some simplifying assumptions. These assumptions are eliminated in PKQuest [5], a new, general-purpose physiologically based pharmacokinetic (PBPK) software routine. The approach used by PKQuest is to first use the IV input data to find the PBPK parameters for ethanol, and then, using these parameters, solve for the time dependent IV input function that would produce a blood time course identical to the time course observed with the orally administered drug. This IV input function is a direct measure of the rate that the oral dose reaches the systemic circulation, i.e. the PA. Using as input just the PBPK parameters and the blood ethanol levels produced by an oral dose, PKQuest outputs (in both tabular and graphical form) the time course of the intestinal absorption and the PA (see [5] for the mathematical and computational details).

The difficulty in defining the FPM of ethanol has created a controversy over the question of the magnitude of ethanol metabolism that occurs in the stomach before reaching the liver [1]. It has been observed that the "FPM" is significantly reduced when ethanol is infused intraduodenally compared to the standard oral administration and this difference has been interpreted as representing gastric mucosal metabolism [6-8]. Clearly, if there are errors in the measurement of FPM then this estimate of gastric metabolism may be spurious. In addition to providing a value for FPM, PKQuest also provides a direct measurement of the rate and amount of the orally administered solute that reaches the liver. In this paper, PKQuest will be ap- 
Table I: Organ Weights and Blood Flows for Standard Human (70 Kg, $20 \%$ Fat)

\begin{tabular}{lll}
\hline Organ & Weight $\mathbf{K g})$ & Blood Flow (lit/min/Kg) \\
\hline & & \\
Artery & 1.1 & --- \\
Vein & 4.4 & --- \\
Liver & 1.8 & 0.25 \\
Portal & 1.5 & 0.75 \\
Kidney & 0.31 & 4.0 \\
muscle & 33.0 & 0.0266 \\
brain & 1.4 & 0.56 \\
heart & 0.33 & 0.8 \\
lung & 0.536 & 14.98 \\
skin & 2.6 & 0.12 \\
fat & 14 & 0.056 \\
"other" & 4.12 & 0.054 \\
"bone" & 4.90. & 0.0 \\
\hline
\end{tabular}

plied to investigations of human ethanol pharmacokinetics, providing information about the FPM; the time course of intestinal absorption, and the total amount of ethanol reaching the liver. This is the first human PBPK model for ethanol incorporating intestinal absorption and FPM. Pistino and Conolly [9] have previously developed a rat PBPK that quantitated ethanol FPM.

The PKQuest program and all the examples used here are freely available on the WEB at [www.pkquest.com].

\section{Methods}

\section{PBPK program and its assumptions}

PKQuest [5] was used for all the analysis in this paper. All the figures shown in this paper are direct copies (in jpeg format) of standard PKQuest output. PKQuest makes several simplifying assumptions (see [5] for details). It is assumed that each organ can be modeled as a single wellstirred compartment. In addition, for the two solutes investigated in this paper $\left(\mathrm{D}_{2} \mathrm{O}\right.$ and ethanol) it is also assumed that the blood-tissue exchange is flow limited so that the unbound water concentration in the tissue is equal to that in the vein leaving the tissue. This is clearly an approximation and effects such as diffusion gradients, countercurrent exchange and heterogeneous organ blood flows [10-12] will produce small deviations from this assumption.

\section{Standard human parameters}

The PBPK parameters used here are identical to those used in all the other applications of PKQuest $[5,13,14]$. The procedures used for choosing and refining these parameters has been described in [5]. Table 1 lists the organ weights $(\mathrm{Kg})$ and blood flows (liters/min/ $/ \mathrm{gg}$ ) for the standard $70 \mathrm{Kg}$ human. The "liver" flow is the hepatic blood flow; "portal" refers to the portal vein blood flow and to the organs drained by the portal vein (stomach, small and large intestine, spleen and pancreas); "bone" refers to the inert component of bone, cartilage etc. in which it is assumed there is no solute distribution or blood flow; and "other" are all the other organs with a flow and weight adjusted to account for the total body weight (70 $\mathrm{Kg}$ ) and the total default cardiac output (6.06 liters/min). These organ weights are for the default value of $20 \%$ body fat. They are scaled depending on the value of "Fat" that is input. For a complete listing of all parameters and constants used in PKQuest, download the file gensolve.mws from the PKQuest web site [www.pkquest.com] and view the routines "standardman()" and "defaultpar()".

\section{Experimental data}

The $\mathrm{D}_{2} \mathrm{O}$ data was obtained from the measurements of the Schloerb et al. [15] of the arterial serum concentration after a 15 second bolus injection. The results are for one experiment on one male subject (J.O., total body water of 48.0 liters, Table $1 \mathrm{I}[15])$. The amount of $\mathrm{D}_{2} \mathrm{O}$ injected was back calculated from the total body water and the concentration after 90 minutes when the $\mathrm{D}_{2} \mathrm{O}$ had equilibrated.

A baseline ethanol PBPK model was developed based on the careful and detailed measurements of Norberg et al. [16] of the simultaneous breath and venous blood concentrations for a 30 minute constant IV ethanol infusion. The concentration calculated from the breath measurements was in good agreement with the direct venous measurements. The breath results were used here since they should be closer to the ideal of an arterial sample then the venous measurements from the cubital vein. The total body water for each subject in this study was directly measured using $\mathrm{D}_{2} \mathrm{O}$. The time course of the mean values 

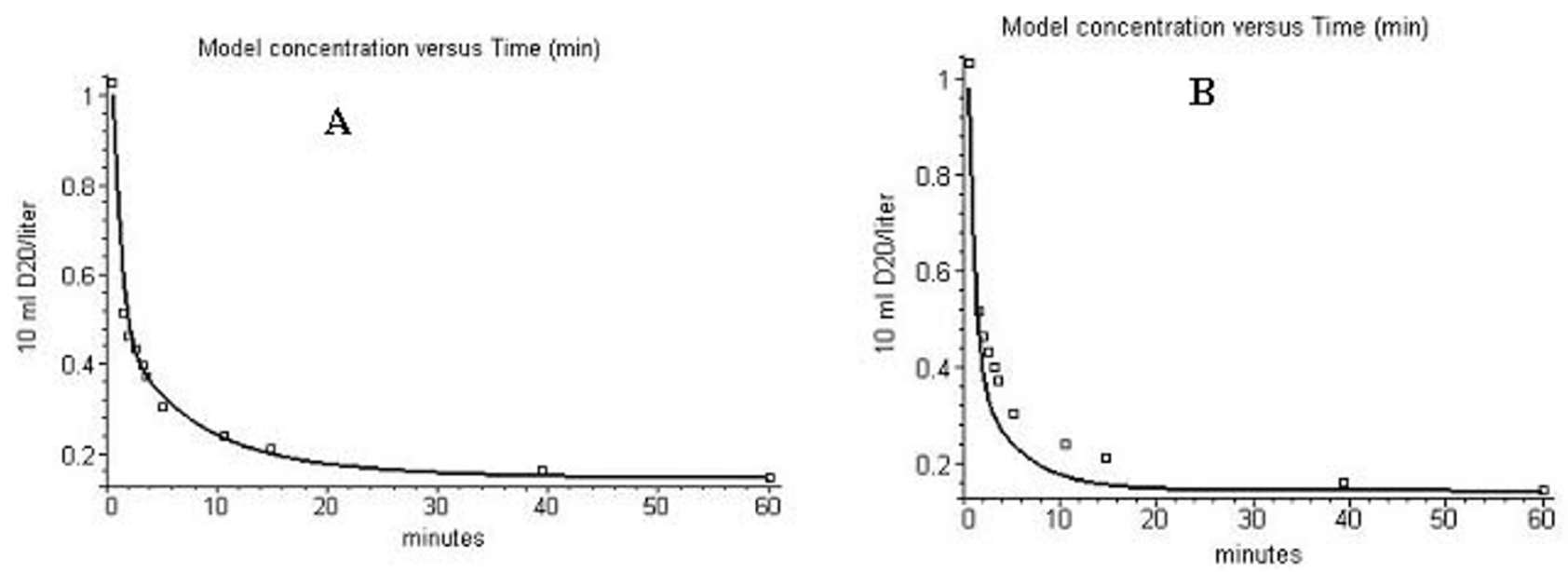

Figure I

Comparison of the time course of the $\mathrm{D}_{2} \mathrm{O}$ arterial blood water concentration (solid line) and the experimental results (squares) of Schloerb et al. [15] using the default PBPK parameters. A) Default muscle blood flow. B) Twice the default muscle blood flow.

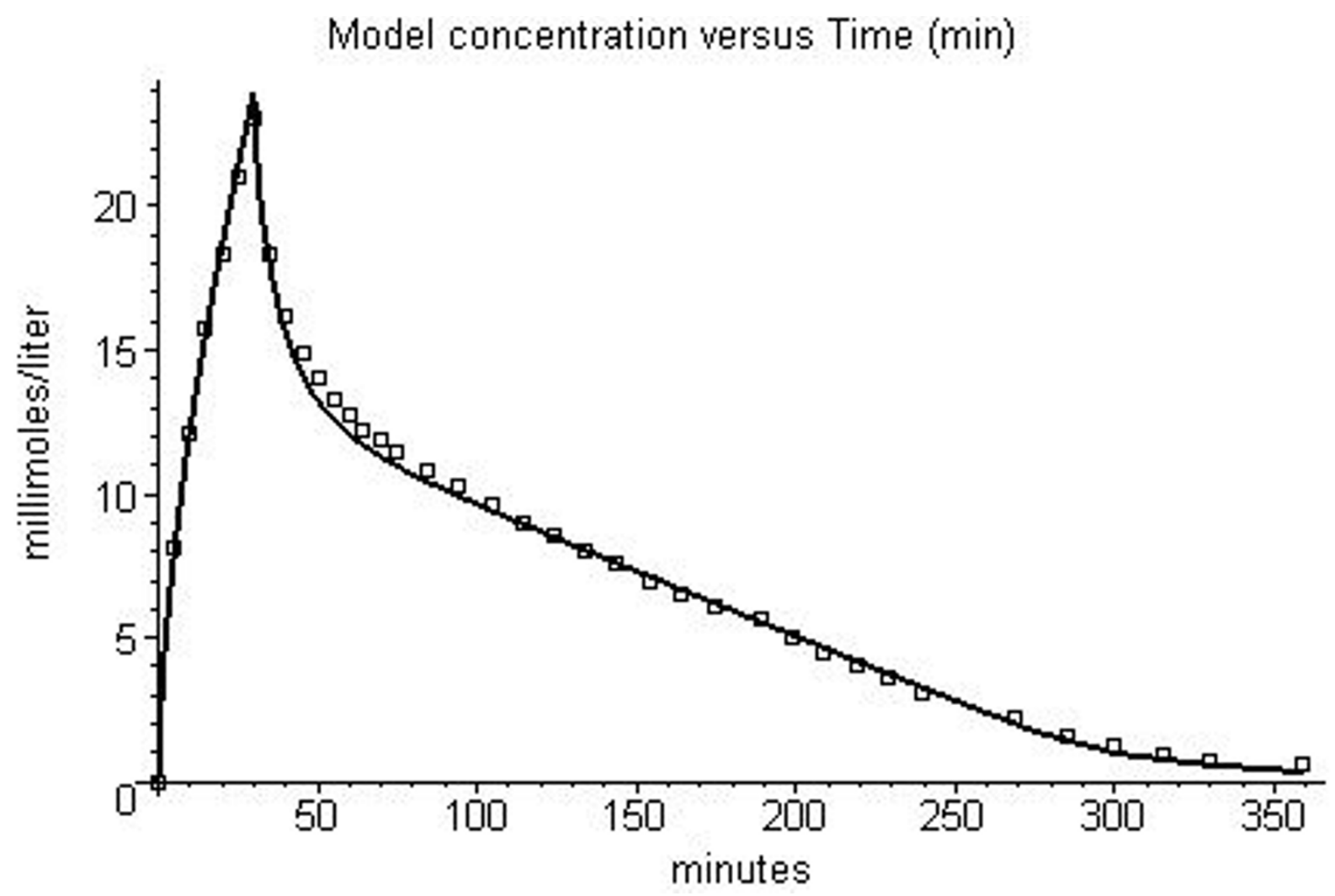

Figure 2

Comparison of the time course of the ethanol arterial blood water concentration (solid line) and the experimental results of Norberg et al. [16]. 


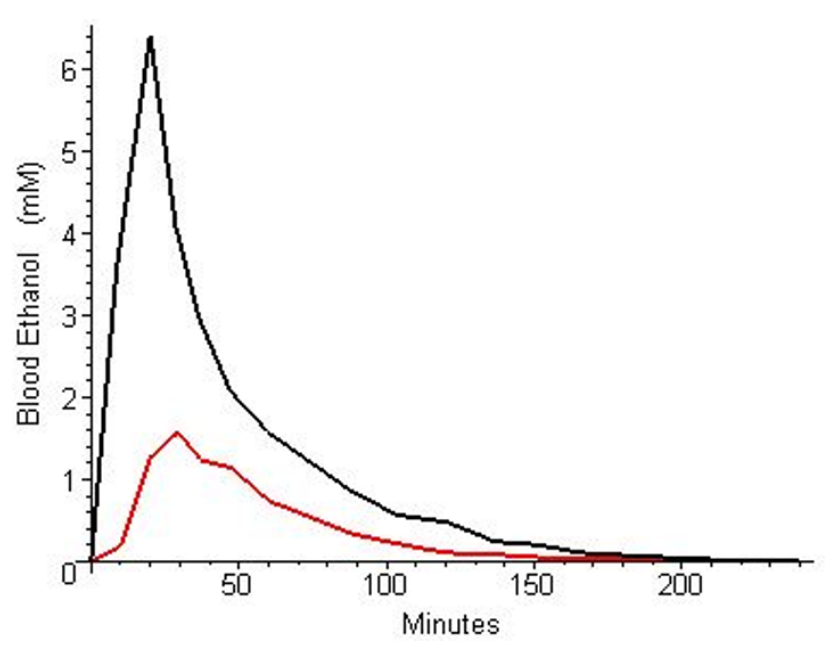

Figure 3

Experimental venous ethanol concentration when a dose of $0.15 \mathrm{gm} / \mathrm{Kg}$ ethanol is administered either IV (black) or orally (red) along with a coincident meal. (Data taken from fig. I of DiPadova et al. [17]).

for all 16 subjects (figure 1, [16]) was modeled using PKQuest. The data of DiPadova et al. [17] was used for the determination of the rate of absorption of oral ethanol, along with calculations of the PA and FPM. PKQuest was applied to the data plotted in figures 1 and 3 of DiPadova et al. [17] which describe the mean blood ethanol for subjects given $0.15 \mathrm{~g} / \mathrm{Kg}$ body weight (fig. 1) or $0.3 \mathrm{~g} / \mathrm{Kg}$ (fig. 3 ) either orally (with or without a breakfast) or IV on alternate days.

The experimental data points were obtained by using UNSCAN-IT (Silk Scientific Corporation) to read the data from the published figures.

\section{Results}

\section{Determination of baseline resting muscle blood flow}

The baseline resting muscle blood flow used in PKQuest was determined by fitting the $\mathrm{D}_{2} \mathrm{O}$ data of Schoerb et al. [15] in which the arterial $\mathrm{D}_{2} \mathrm{O}$ concentration was measured after a 15 second venous injection of $\mathrm{D}_{2} \mathrm{O}$. As described previously [5], each compound modeled by PKQuest is characterized by a short Maple [www.maplesoft.com] procedure that lists the model parameters that are unique for that solute. The procedure for $\mathrm{D}_{2} \mathrm{O}$ is very simple because it corresponds to the default case so that very few parameters have to be input:

d20_schoerb:=proc()

defaultpar():

Wtot:=81.9;
standardhuman(Wtot);

cunit:="10 ml D20"; \# $10 \mathrm{ml} /$ liter is equivalent to vol\%

concunit [artery] :=3; input data is concentration in free water

ninput:=1; finput[1]:

table $([$ organ $=$ vein, type $=1, \quad$ rate $=27.6, \quad$ tbeg $=0$, tend $=.25$, $\operatorname{csteady}=0$, padjust $=0]$ );

end proc:

The published data was for subject JO (body weight 81.9 $\mathrm{Kg}$ and total body water $=48$ liters). This value for total body water ( 0.58 liter $/ \mathrm{Kg}$ ) corresponds to a body fat fraction of about 0.2 [18].. This subject was defined as the "standardhuman" (Fat = 0.2). The values of the water fraction parameter for each tissue compartment in PKQuest was then adjusted so that the model total body water equaled this measured value. Since, this man is the "standardhuman", all the PBPK parameter are input simply by calls to the "defaultpar()" and "standardhuman()" procedures. All units in PKQuest are in terms of liters, Kg and minutes. The parameter "cunit" is the amount unit (concentration $=$ cunit/liter). Since the $\mathrm{D}_{2} \mathrm{O}$ concentration was expressed in vol\%, this corresponds to a cunit of $10 \mathrm{ml}$. The only other information that is needed is concunit [artery] $=3$, which indicates that the input data is the arterial free water concentration. The observed equilibrium concentration was $0.144 \mathrm{vol} \%$ which corresponds to a total $\mathrm{D}_{2} \mathrm{O}$ dose of $69.05 \mathrm{ml}$, or $276 \mathrm{ml} / \mathrm{min}$ for 0.25 minutes, or 27.6 units $/ \mathrm{min}$ if 1 unit $=10 \mathrm{ml}$. The output of PKQuest for this data is shown in fig. 1A (all of the figures in this paper are taken unmodified from the standard output of PKQuest). The value for the resting muscle blood flow (0.0266 Lit/min/Kg) was determined by adjusting the flow to optimize the fit to the data using the Powell minimization procedure of PKQuest. The agreement between the PBPK prediction (solid line) and the experimental measurements (squares) is very good.

This agreement is sensitive to the value used for the muscle blood flow. For example, fig. 1B shows the effect of doubling the total muscle blood flow from this default, best fit, value of 0.0266 to 0.0532 liters $/ \mathrm{min} / \mathrm{kg}$ (increasing the cardiac output from 6.06 to 6.93 liters/min for a $70 \mathrm{Kg}$ man). Muscle blood flow can vary many fold and one would expect that it would depend on the specific experimental conditions, such as whether the subject was supine or ambulatory. In any case, muscle blood flow is routinely varied when fitting data with PKQuest. This variation may have some physiological significance and/or it may be just a "fudge factor". 


\section{Standard Ethanol PBPK parameters}

The recent measurements of Norberg et al.[16] of the time course of ethanol blood levels after an IV input were used to determine the standard ethanol PBPK parameters. PKQuest was used to model the time course of the mean blood values of the 16 subjects ( 8 men, 8 women). This data was expressed as the concentration in the blood in units of millimoles/liter of blood water. Both breath and direct venous sampling results were reported and they were in very good agreement. The results shown in fig. 2 use the breath measurements since they should be closer to the ideal of an arterial sample then the venous measurements from the cubital vein. The PKQuest procedure that is used for ethanol is listed below:

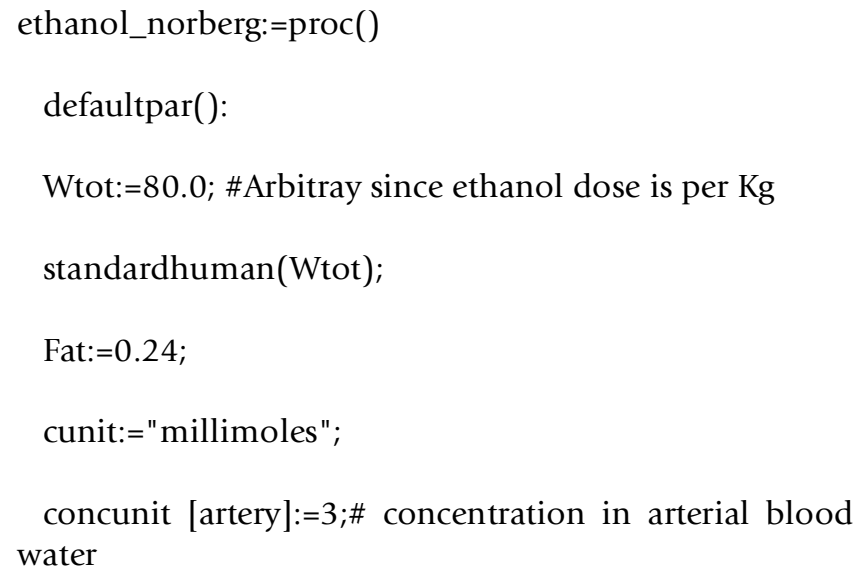
water

$$
\text { Vm [liver]:=2.1: }
$$

Km [liver]:=0.04;

fw [lipid]:=1.0/0.074; \# = 1/(oil-water partition coefficient for ethanol)

$$
\text { ninput:=1;finput[1]: }
$$

table $($ organ $=$ vein, type $=1$, rate $=23.15$, tbeg $=0$, tend $=30$, csteady $=0$, padjust $=0]$ );

end proc;

The value for Fat (fraction of body weight that is fat) of 0.24 was chosen so that the model subject had a water volume equal to the average $\mathrm{D}_{2} \mathrm{O}$ volume of distribution of the 8 males and 8 females in the study (44.5 liters for 80 Kg human). The muscle blood flow was the same as the (default) value that was used in fig. $1 \mathrm{~A}$ for the $\mathrm{D}_{2} \mathrm{O}$ data. Since liver metabolism accounts for greater than $95 \%$ of ethanol removal, it is assumed that the liver is the only site of ethanol elimination [19]. The liver metabolic constants $(\mathrm{Vm}=2.1 \mathrm{millimoles} / \mathrm{min}$ for $80 \mathrm{Kg}$ man, $\mathrm{Km}=0.04 \mathrm{mM})$ were adjusted using the Powell minimization procedure of PKQuest to give the best fit to the data. These values refer to the free water concentration in the liver (see [5] for details). There are a number of isoenzymes of alcohol dehyrogrenase $(\mathrm{ADH})$ in the human liver and this model $\mathrm{Km}$ of $0.04 \mathrm{mM}$ is consistent with the in vitro $\mathrm{Km}$ of 0.048 $\mathrm{mM}$ observed for one of these isoenzyme [20]. It is assumed that ethanol distributes in the total body water, which is the default in PKQuest. The parameter fw [lipid] $=1 / 0.074$ represents the inverse of the oil/water partition coefficient and indicates that the equilibrium ethanol concentration in the body fat is $7.4 \%$ of the concentration in body water [21]. The ethanol dose was $0.4 \mathrm{gm} / \mathrm{Kg}$, given as a constant 30 minute infusion, corresponding to an infusion rate of 23.15 millimoles/min for an $80 \mathrm{Kg} \mathrm{hu}$ man. This Maple procedure (ethanol_norberg) completely specifies the PBPK parameters. All the other model parameters are set by the calls to defaultpar() and standardhuman(). Figure 2 shows the output of PKQuest for this input. Again, the agreement between the PBPK prediction for the arterial blood concentration (solid line) and the observed breath data (squares) is quite good.

\section{Quantitation of ethanol first pass metabolism (FPM)}

DiPadova et al. [17] measured the blood ethanol in subjects that were given the same dose of ethanol, either IV (20 minute constant infusion) or orally (over $10 \mathrm{~min}$ utes), on consecutive days. Figure 3 shows the venous concentrations as a function of time when a low dose of ethanol $(0.15 \mathrm{~g} / \mathrm{Kg}$, equivalent to approximately $12 \mathrm{oz}$ of beer) was given at the same time as a standard breakfast either IV (black) or orally (red). The area under the oral dose curve is only $27 \%$ of that for the IV dose. This would correspond to a FPM of $73 \%$ if metabolism was linear i.e. only $27 \%$ of the orally ingested ethanol reaches the systemic circulation.. As will be shown below, the actual FPM is much less than this.

The data in fig. 3 was analyzed using PKQuest. Figure 4A shows the agreement between the PBPK model and the venous plasma ethanol concentration for the IV input data. The PBPK parameters used in this calculation were identical to those used for figure 2 except that the portal blood flow was increased $50 \%$ because of the coincident meal [22-24], the liver Vm was increased from 2.1 to 2.5 because of the induced ethanol effect $[25,26]$ and the muscle blood flow was doubled. Also, the parameter "concunit [vein]" was set $=2$, indicating that the whole blood venous concentration was used as the input data.

Figure 4B shows the output of PKQuest when the "findabsorption" [5] option was applied to the oral ethanol input data. Since the IV and oral data used for figs. $4 \mathrm{~A}$ and $4 \mathrm{~B}$ were collected in the same subjects under identical conditions, exactly the same PBPK parameters where used for both calculations. The open squares show the time course 

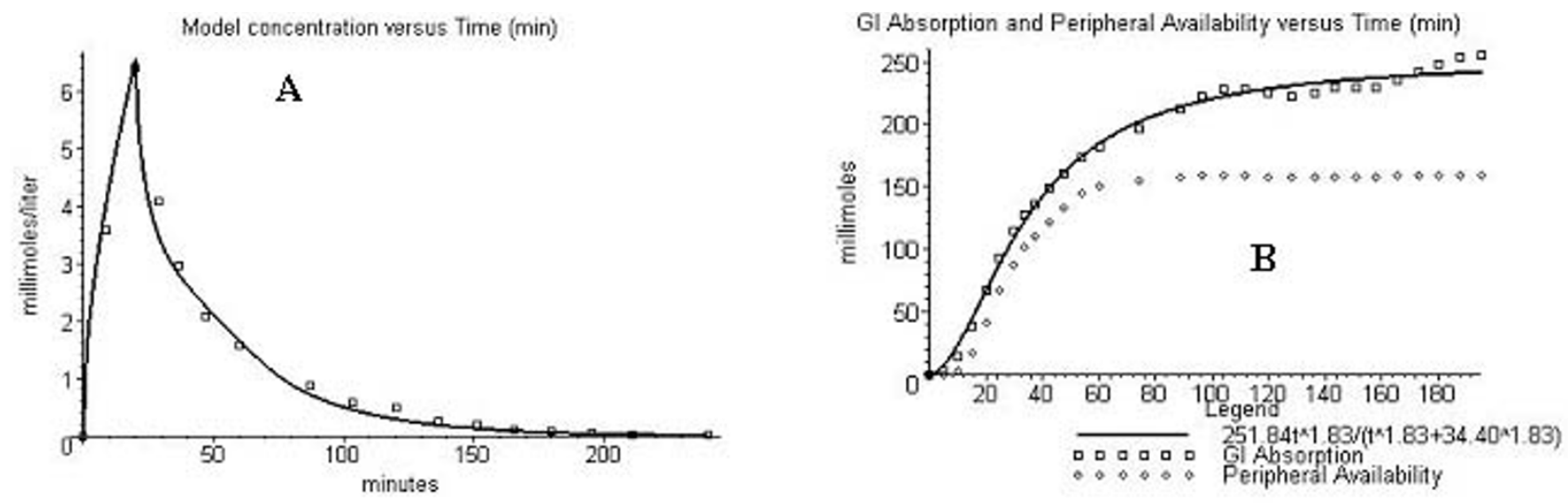

\section{Figure 4}

PKQuest predictions for an IV or oral input of $0.15 \mathrm{gm} / \mathrm{Kg}$ coincident with a standard meal. A) Comparison of the model time course of the ethanol venous whole blood concentration (solid line) and the experimental results (squares) of DiPadova et al. [I7] for the IV input. B) Predicted total ethanol intestinal absorption (squares) and peripheral availability (diamonds) for the oral dose. The solid line is a 3 parameter approximation to the model absorption data.

of the predicted GI ethanol absorption (amount entering the liver in the portal vein [5]). The solid line is a 3 parameter approximation to the absorption data (absorption at time $\mathrm{t}=\mathrm{A}\left[\mathrm{t}^{\mathrm{H}} /\left(\mathrm{t}^{\mathrm{H}}+\mathrm{T}^{\mathrm{H}}\right)\right]$ where $\mathrm{A}=$ total absorption, $\mathrm{H}=$ Hill exponent, $\mathrm{T}=$ half time).

It can be seen that the intestinal absorption (fig. $4 \mathrm{~B}$ ) is complete within about 2 hours. The total predicted absorption of 250 millimoles is slightly less than the actual total oral dose of 260 millimoles. Although this is consistent with a small amount of gastric mucosal metabolism (10 millimoles) it is probably not significant. The calculation of the total absorption for this low dose is critically dependent on the accuracy of the venous blood measurements at long times. Since the liver has such a low $\mathrm{Km}$ (0.04 $\mathrm{mM})$, significant amounts of intestinal absorption could be occurring at long times even though the venous concentration remained close to zero because of the high rate of liver clearance. The fluctuation in the total absorption points at long times in fig. $4 \mathrm{~B}$ is caused by errors in these long time blood measurements.

When the "findabsorption" option is used, PKQuest also routinely calculates the time course of the "Peripheral Availability" (PA), indicated by the diamonds in fig. 4B The PA is defined as the equivalent IV input that would produce the observed venous concentration. Thus, an IV input with the time course of the diamonds in fig. $4 \mathrm{~B}$ would produce exactly the same blood concentration as a function of time as the GI absorption represented by the open squares. PA is related to FPM by the relation: FPM = Total Dose - PA. Thus for the data used for fig. 4B, the PA of 160 millimoles corresponds to a FPM of 100 millimoles (total dose $=260$ ). This represents $38 \%$ of the total dose, about half the value of $73 \%$ using the classical AUC approach.

The FPM for this data was also estimated by using the one compartment approach [3]. Figure 5A shows the optimized one compartment Michaelis-Menten fit to the tail of the venous ethanol data $(\mathrm{Vm}=8.68$ millimoles $/ \mathrm{min}$; $\mathrm{Km}=5.46 \mathrm{mM})$. Figure $5 \mathrm{~B}$ shows the corresponding fit to the data of DiPadova etal. [17] for a doubling $(0.3 \mathrm{gm} / \mathrm{Kg})$ of the oral dose (see below). Not surprisingly, a one compartment model does not provide an accurate fit to the ethanol data. The fit is better for the large input dose. Using these values of $\mathrm{Vm}$ and $\mathrm{Km}$, the PA was determined for the IV and oral dose by assuming a one compartment model and integrating the liver metabolism $(=\mathrm{VmC} /$ $(\mathrm{Km}+\mathrm{C})$ where $\mathrm{C}$ equals venous blood concentration) over the time. For the data of fig. 4, this one compartment method yielded a FPM of 59\%. This is less than the $73 \%$ estimated from the AUC, but significantly greater than the 38\% using PKQuest.

DiPadova et al.[17] also measured the blood levels resulting from an IV or oral input of a larger ethanol dose $(0.3$ $\mathrm{gm} / \mathrm{Kg}$, total dose of 520 millimoles for the $80 \mathrm{Kg}$ model subject) and this time they also compared the fasting and standard breakfast case. Figures $6 \mathrm{~A}$ and $6 \mathrm{~B}$ show the PKQuest output for the IV and oral data when the ethanol was taken with the same standard meal as was used for the data in figs. $4 \mathrm{~A}$ and $4 \mathrm{~B}$. The parameters were identical to those used in figs. 4 except that the liver $\mathrm{Vm}$ was reduced from 2.5 to 2.4 (a different set of subjects were used in the two sets of experiments). It can be seen from fig. $6 \mathrm{~B}$ that, for this higher dose, the total peripheral availability (diamonds) is only about 40 millimoles less than the total 

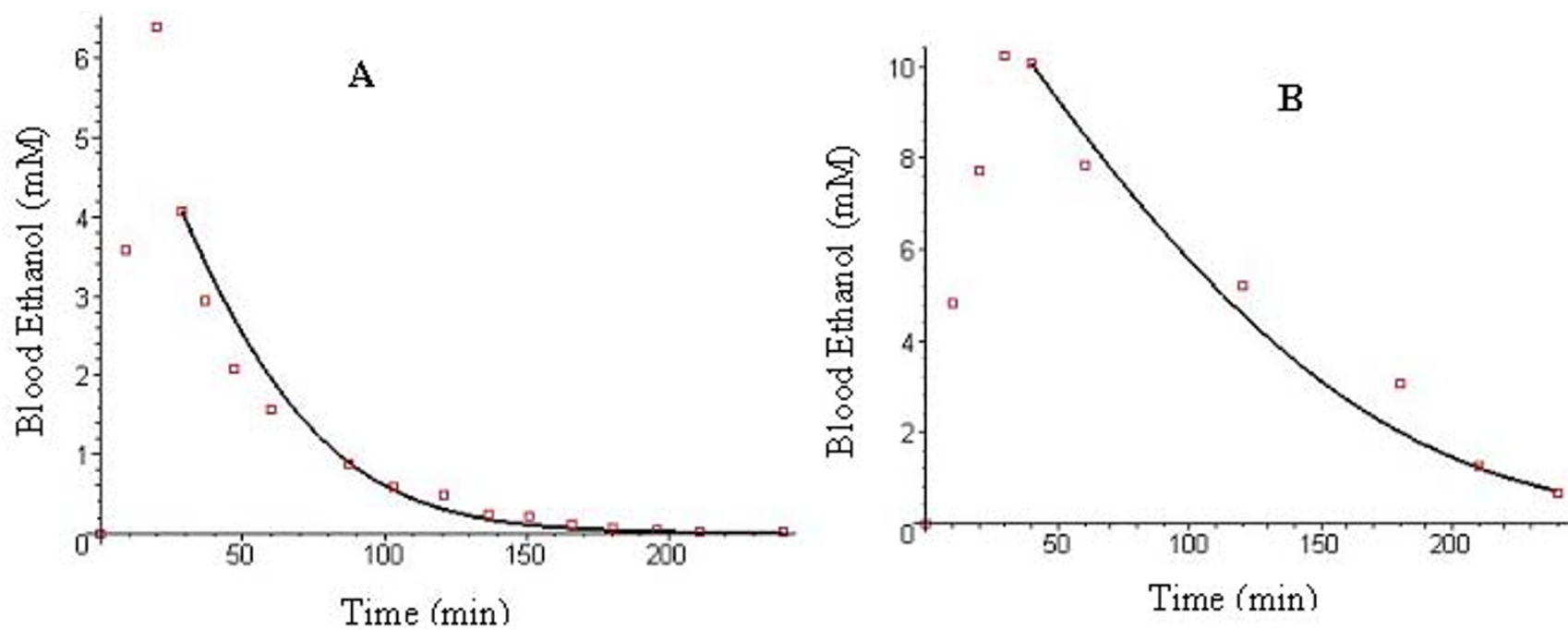

Figure 5

Optimized one compartment fit to the tail of the venous blood curve for IV ethanol. A) IV input of $0.15 \mathrm{gm} / \mathrm{Kg}$. B) IV input of $0.3 \mathrm{gm} / \mathrm{Kg}$.
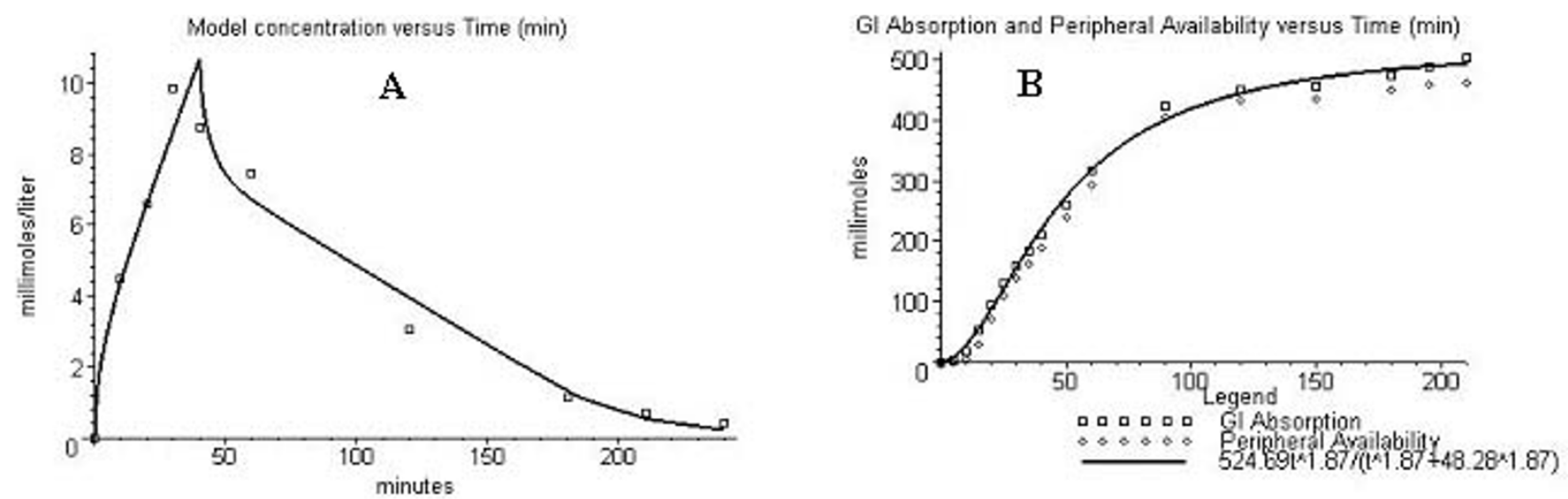

Figure 6

Similar to fig. 4 except that the IV dose was $0.3 \mathrm{gm} / \mathrm{Kg}$.

dose of 520 millimoles, corresponding to a fractional FPM of $7 \%$. This is much less than the FPM value of $44 \%$ determined by using the AUC approach or of $30 \%$ using the one compartment approach. The time course of the intestinal absorption for the two doses (compare fig. $4 \mathrm{~B}$ and fig. 6B) has the same shape (e.g. same Hill coefficient) but occurs over a slightly longer time for the larger dose (Hill half time $=34$ minutes for $0.15 \mathrm{gm} / \mathrm{Kg}$ dose, and $48 \mathrm{~min}$ utes for $0.3 \mathrm{gm} / \mathrm{Kg}$ dose) presumably because the larger oral dose was ingested over a longer time (20 minutes for $0.3 \mathrm{gm} / \mathrm{Kg}$ versus 10 minutes for $0.15 \mathrm{gm} / \mathrm{Kg}$ ).

Figures 7A and 7B show the PKQuest output for the same ethanol dose $(0.3 \mathrm{gm} / \mathrm{Kg})$ given to fasting subjects. The PBPK parameters for these experiments had the portal blood flow reduced back to the default fasting value. Also, the liver Vm was reduced back to 2.0, the same value that was used for the fasting data of Norberg et al [16] in fig 2. This change in ethanol metabolism is consistent with earlier observations that the rate of ethanol disappearance from the blood is increased by a coincident meal $[25,26]$. It can be seen in fig. 7B that the PA (diamonds) is nearly identical to the GI absorption (squares) so that FPM is close to zero for the fasting subjects. The AUC and one compartment approach also indicate a small FPM for this case. 

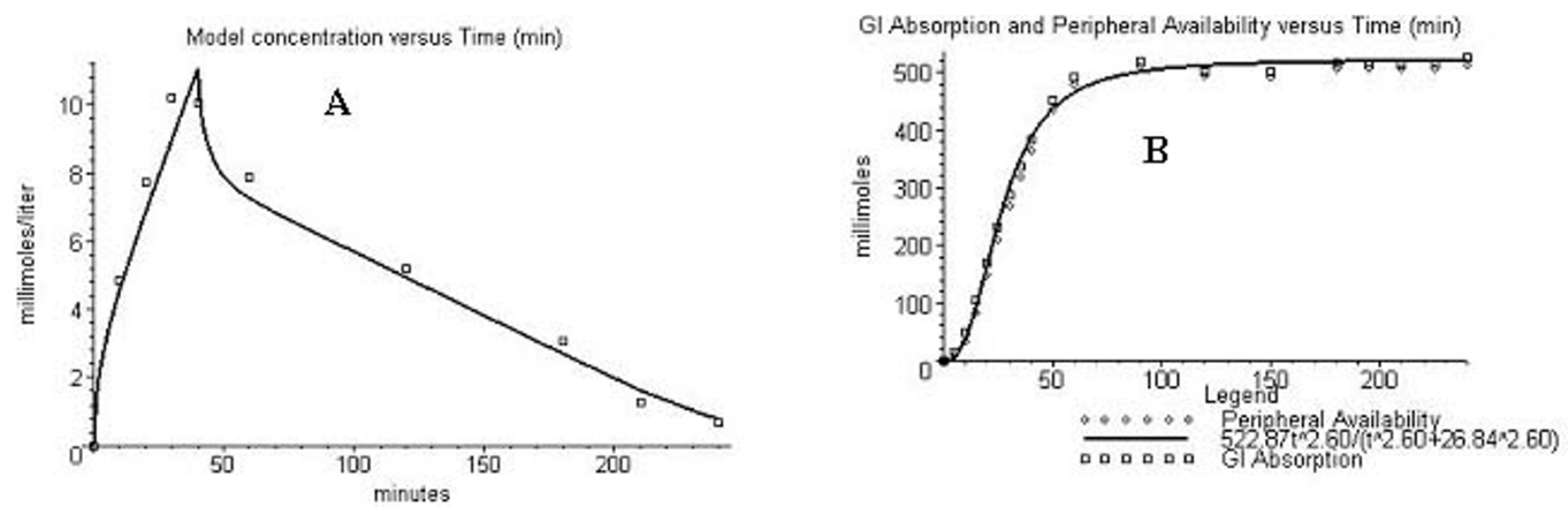

Figure 7

Similar to fig. 6 except that the subjects were fasting.

\section{Discussion and conclusions Validity of the PBPK model}

The philosophy behind the use and development of PKQuest differs from that of the standard PBPK applications. PBPK models have usually been applied to animal data where it is possible to accurately measure and control the relevant parameters (tissue distribution, organ blood flows, etc.). In contrast, for human studies, not only are the PBPK parameters poorly characterized, but they are subject to large individual variation. The impetus behind PKQuest is to try and develop a "standard" set of human PBPK parameters that can be applied in the absence of this detailed information. For this reason, the application of PKQuest to human data should be regarded as a first approximation.

Since muscle represents roughly $50 \%$ of the body mass, the muscle blood flow, which can vary 10 fold or more depending on experimental conditions, is the single most important PBPK parameter and the parameter that limits the accuracy of the PBPK model. The pharmacokinetics of $\mathrm{D}_{2} \mathrm{O}$ (or other tracers of water) are especially simple since the rate of elimination is very slow and it distributes simply in the organ water. As shown by figs. $1 \mathrm{~A}$ and $1 \mathrm{~B}$, the $\mathrm{D}_{2} \mathrm{O}$ kinetics are sensitive to the muscle blood flow and the data of Schloerb et al. [15] was used to determine the resting muscle blood flow value (0.0266 liters/min/Kg) for the "standardhuman". Although it is problematical to base such an important parameter on just one set of measurements on one subject, this represents the only published measurement of this type that I could find. It is also an approximation to treat the muscle as a single compartment with a uniform blood flow and in some human PBPK models [27] two muscle compartments ("resting" and "working") are included. For the PKQuest modeling, the muscle blood flow is usually treated as an adjustable parameter and not as a strictly determined PBPK constant.
The ethanol data that was modeled are the mean values, averaged over a number of individuals. Because of the non-linear nature of the pharmacokinetics, it is an approximation to treat these mean values as representative of an equivalent "average" human [28]. However, it should provide a first approximation to the PBPK parameters of an "average" human. For the common case where the published mean values are the only data that is available, there is no other option.

In the PKQuest modeling of ethanol, it is assumed that ethanol distributes freely in the tissue water. This seems like a reasonable assumption given the physical-chemical properties of ethanol. Measurements of plasma/whole blood concentration ratios show that this assumption is valid for red blood cells [29]. However, Norberg et al. $[16,19]$ has shown that the ethanol volume of distribution estimated using a 2 compartment model is significantly less than the total water volume and they have reviewed the literature that suggests that the tissue ethanol distribution may be restricted. Despite this, the agreement between PKQuest results and the Norberg et al. data (figure 2) indicate that this assumption is a useful approximation given the limitations of using the mean data.

\section{Ethanol first pass metabolism and intestinal absorption}

The definition of FPM implemented in PKQuest is both rigorous and intuitive. It is based on defining a time dependent "peripheral availability" (PA) that is equal to the rate of IV input that would produce the observed time course of the plasma ethanol concentration. This PA is a direct quantitative measure of the amount of ethanol that has reached the systemic circulation. The FPM is then defined as the difference between the total dose administered and the PA. The classical procedure of determining FPM from the AUC (eq.1) markedly overestimates the FPM. For the case of the small ethanol dose $(0.15 \mathrm{gm} / \mathrm{Kg})$ 


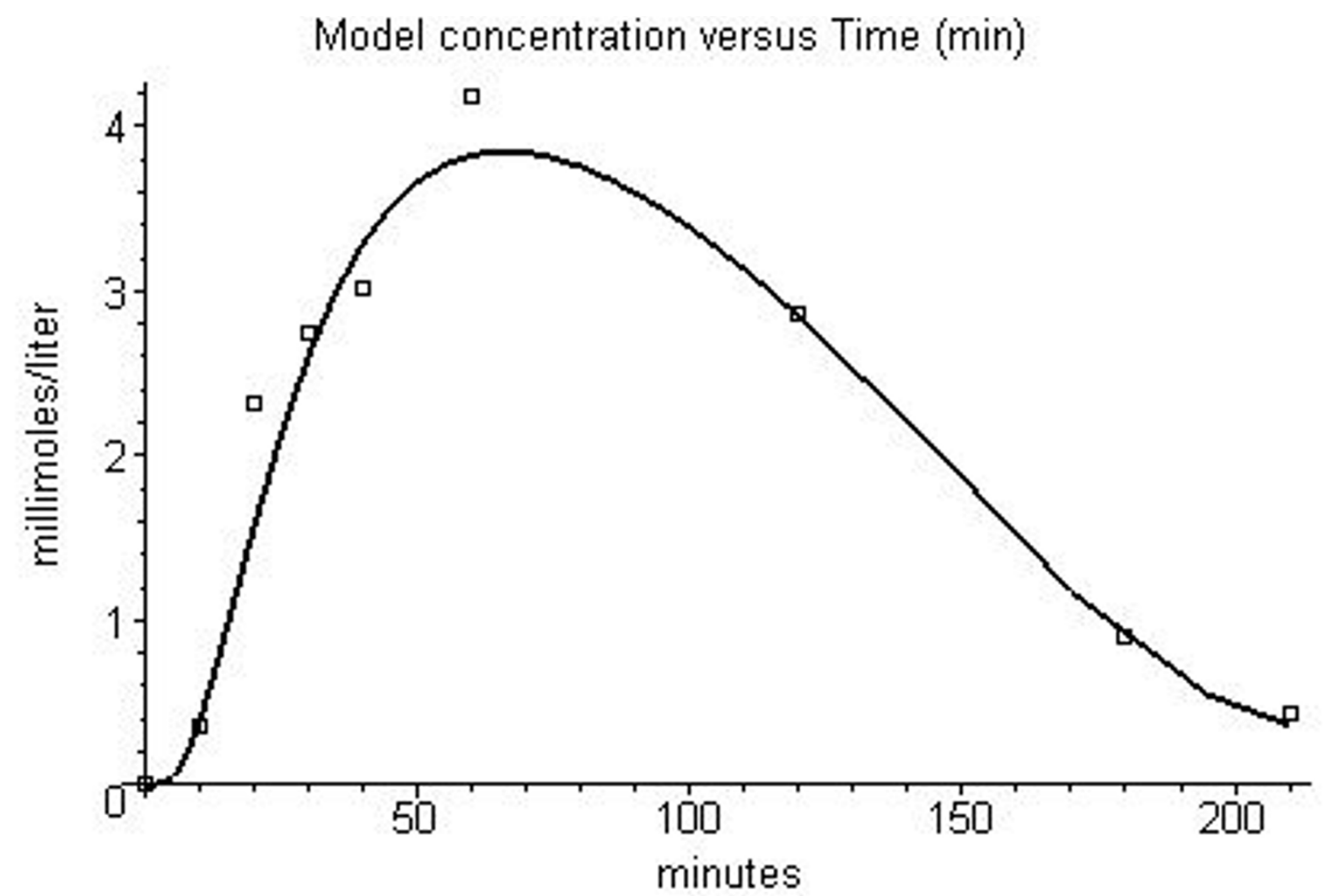

Figure 8

Comparison of PKQuest prediction of venous blood level (line) versus the experimental data for an oral dose of 0.15 gm/Kg. The PKQuest intestinal absorption was described by the 3 parameter Hill equation obtained in fig. 6.

taken with a meal, the AUC estimate of fractional FPM of $73 \%$ was about twice the value $(38 \%)$ obtained using PKQuest. For the higher dose $(0.3 \mathrm{gm} / \mathrm{Kg})$ the error was even larger (44\% versus $7 \%$ ). Although the more recent one compartment approximation provides a better estimate of FPM, it is still significantly larger than the PKQuest values (59\% versus 38\% for $0.15 \mathrm{gm} / \mathrm{Kg}$ dose and $30 \%$ versus $7 \%$ for $0.3 \mathrm{gm} / \mathrm{Kg}$ dose).

The error in both these approximations to FPM results primarily from the fact that the systemic appearance of the oral ethanol taken with a meal is slow compared to the 20 minute constant infusion that was used to obtain the IV AUC. Since the liver ethanol metabolism saturates at low concentrations, the shorter dose will have a higher average blood concentration and a corresponding lower value of fractional metabolism. The error would be less if the time course of the oral absorption was similar to that of the IV input. This explains why the AUC calculated FPM [17] was greatly reduced when the subjects were fasting so that the gastric emptying rate was increased and GI absorption was more rapid (compare figs. $6 \mathrm{~B}$ and $7 \mathrm{~B}$ ) and closer to the rate of IV infusion. The critical dependence of the AUC on the rate of systemic ethanol input was first pointed out by
Wagner [30]. He showed that the AUC for the oral input was equal to (or even larger than) the IV AUC if the IV infusion was administered at a slower rate (for 2 hours) than the rate of intestinal absorption.

Failure to appreciate the problems inherent in using the AUC or one compartment method to measure FPM has led to the concept that the gastric mucosa metabolizes a significant fraction of oral ethanol. Lieber and colleague observed that the "FPM" was reduced when ethanol was infused intraduodenally compared to the standard oral administration and concluded that the difference must have resulted from gastric mucosa metabolism [6,7]. This decreased FPM for the intradudenal dose is probably a result of the much more rapid time course of absorption when the ethanol is administered directly into the duodenum. PKQuest provides a direct estimate of the amount of ethanol reaching the liver. As shown by figs. 4B, 6B and $7 \mathrm{~B}$, the total amount of absorbed ethanol reaching the liver does not differ significantly from the total oral dose, indicating that gastric mucosa metabolism is negligible.

In addition to providing a direct measure of PA and FPM, PKQuest also provides, for the first time, quantitative in- 

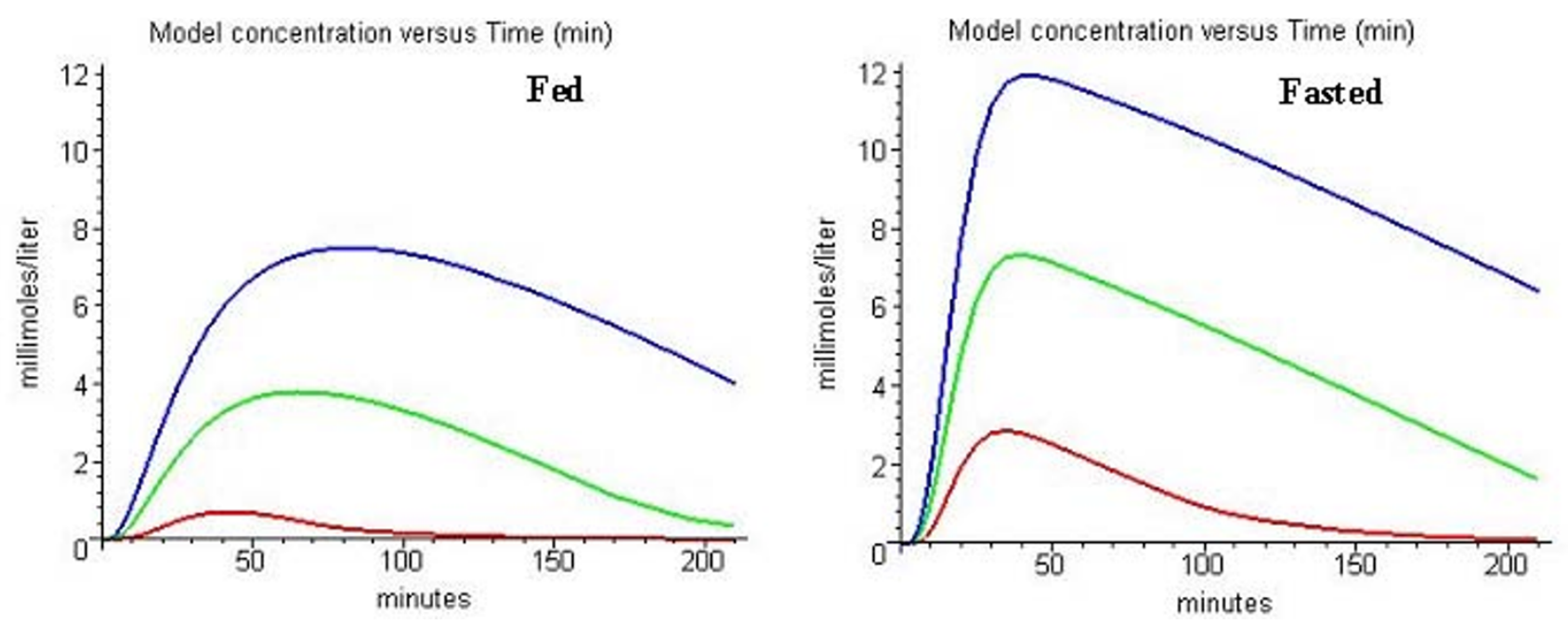

Figure 9

Predicted venous ethanol concentration for an oral intake of I (red), 2 (green) or 3 (blue) cans of beer over a 20 minute period. A) Coincident with a standard meal. B) With no other food.

formation about the rate of intestinal absorption of an orally administered drug that has non-linear metabolism. A comparison of figs. $6 \mathrm{~B}$ and $7 \mathrm{~B}$ provides a dramatic view of the decreased rate of ethanol absorption when it is accompanied by a meal. In the fasting state, ethanol is almost completely absorbed within 50 minutes (fig. 7B) while the ethanol administered with a standard breakfast takes 3 or more hours for complete absorption (fig. 6B). Levitt et al.[31] simultaneously measured the rate of human gastric emptying and ethanol blood concentration with and without a meal and concluded that the rate of ethanol absorption was primarily limited by the rate of gastric emptying. The similarity between the time course of ethanol absorption seen in figs. $6 \mathrm{~B}$ and $7 \mathrm{~B}$ and the gastric emptying time course observed by Levitt, et al. [31] supports this conclusion.

\section{Simulation of ethanol blood levels after oral intake}

Another feature of PKQuest is that it provides a simple interface that can be used to make quantitative predictions of the blood levels produced by different oral doses. As shown in figs. $4 \mathrm{~B}, 6 \mathrm{~B}$ and $7 \mathrm{~B}$, PKQuest routinely fits the time course of the intestinal absorption with a 3 parameter Hill type equation (Absorption at time $\mathrm{t}=\mathrm{A}\left[\mathrm{t}^{\mathrm{H}} /\left(\mathrm{t}^{\mathrm{H}}\right.\right.$ $\left.+\mathrm{T}^{\mathrm{H}}\right)$ ] where $\mathrm{A}=$ total absorption, $\mathrm{H}=$ Hill exponent, $\mathrm{T}=$ half time). This 3 parameter input function can then be used as the input into PKQuest. For example, the intestinal absorption input function that was determined for the $0.3 \mathrm{gm} / \mathrm{Kg}$ dose of ethanol ingested with a meal (see fig. $6 \mathrm{~B})$ can be used for a model simulation using the following settings for finput: table $([$ organ $=$ liver, type $=3$, rate $=524$, tbeg $=0$, tend $=48.28$, $\mathrm{hn}=1.87$, csteady $=0$

This corresponds to a total input of 524 millimoles into the portal vein ("organ = liver") with a Hill coefficient ("hn") of 1.87 and a half time ("tend") of 48.28 minutes. Figure 8 shows that the simulated blood level for this input function (solid line) slightly underestimates the peak value, but otherwise is in good agreement with the experimental data (squares) that was used to determine this input function. In Figure 9 this feature is used to illustrate the dramatic effect of a concomitant meal on the blood levels resulting from an oral intake. The figure shows the blood levels for an oral intake of 260 millimoles of ethanol (red line, roughly equivalent to one 12 ounce can of beer); and 2 (green) or 3 (blue) times this dose, all ingested for a 20 minute time period either with ("fed") or without ("fasted") a meal. The model results for the "fed" case (fig. 9, left) used the above rate of intestinal absorption determined from the data in fig. 6B. The "fasted" case (fig. 9 , right) used the intestinal absorption rate determined for the fasting data in fig. $7 \mathrm{~B}$ (Hill coefficient $=2.6$; half time $=26.84$ minutes). In addition, the corresponding values of liver $\mathrm{Vm}$ and portal blood flow for the fed and fasting conditions are used in this simulation. The influence of a meal on ethanol blood levels is especially dramatic at the low intake dose, decreasing the peak blood level by about 4 fold.

\section{Competing interests}

None declared.

ninput:=1;findabs:=0;finput[1]:= 


\section{References}

I. Levitt MD, Levitt DG: Appropriate use and misuse of blood concentration measurements to quantitate first-pass metabolism. J Lab Clin Med 2000, I 36:275-80

2. Levitt MD, Levitt DG: Use of a two-compartment model to assess the pharmacokinetics of human ethanol metabolism. Alcohol Clin Exp Res 1998, 22:1680-8

3. Gentry RT, Sharma R, Lim RT, Baraona E, DiPadova C, Lieber CS: A new method to quantify first pass metabolism of alcohol: Application to the effects of $\mathrm{H2}$-blockers on alcohol bioavailability. Gastroenterology 1992, 92:A8I I

4. Baraona E, Abittan CS, Dohmen K, Moretti M, Pozzato G, Chayes ZW, Schaefer C, Lieber CS: Gender differences in pharmacokinetics of alcohol. Alcohol Clin Exp Res 200I, 25:502-7

5. Levitt DG: PKQUEST: A general physiologically based pharmacokinetic model. Introduction and application to propranolol. BMC Clinical Pharmacology 2002, 2:5

6. Caballeria J, Frezza M, Hernandez-Munoz R, DiPadova C, Korsten MA, Baraona E, Lieber CS: Gastric origin of the first-pass metabolism of ethanol in humans: effect of gastrectomy. Gastroenterology 1989, 97:। 205-9

7. Julkunen RJ, Di Padova C, Lieber CS: First pass metabolism of ethanol - a gastrointestinal barrier against the systemic toxicity of ethanol. Life Sci 1985, 37:567-73

8. Ammon E, Schafer C, Hofmann U, Klotz U: Disposition and firstpass metabolism of ethanol in humans: is it gastric or hepatic and does it depend on gender? Clin Pharmacol Ther 1996, 59:50313

9. Pastino GM, Conolly RB: Application of a physiologically based pharmacokinetic model to estimate the bioavailability of ethanol in male rats: distinction between gastric and hepatic pathways of metabolic clearance. Toxicol Sci 2000, 55:256-65

10. Lifson N, Levitt DG, Griffen WO Jr, Ellis CJ: Intrahepatic distribution of hepatic blood flow: double-input studies. Am J Physiol | 970, 2 | 8: | 480-8

II. Griffen WO Jr, Levitt DG, Ellis CJ, Lifson N: Intrahepatic distribution of hepatic blood flow: single-input studies. Am J Physiol 1970, 2 1 8: 1474-9

12. Doolette DJ, Upton RN, Grant C: Diffusion-limited, but not perfusion-limited, compartmental models describe cerebral nitrous oxide kinetics at high and low cerebral blood flows. J Pharmacokinet Biopharm 1998, 26:649-72

13. Levitt DG: PKQUEST: Capillary Permeability Limitation and Plasma Protein Binding - Application to Human Inulin, Dicloxacillin and Ceftriaxone Pharmacokinetics. Submitted: BMC Clinical Pharmacology. 2002

14. Levitt DG: PKQUEST: Volatile Solutes - Application to Enflurane, Nitrous Oxide, Halothane, Methoxyflurane and Toluene Pharmacokinetics. BMC Anesthesiology 2002, 2:5

15. Schloerb PR, Friis-Hansen BJ, Edelman IS, Solomon AK, Moore FD: The measurement of total body water in the human subject by deuterium oxide dilution. J Clin Invest 1950, 29:1296-310

16. Norberg A, Sandhagen B, Bratteby LE, Gabrielsson J, Jones AW, Fan $H$, Hahn RG: Do ethanol and deuterium oxide distribute into the same water space in healthy volunteers? Alcohol Clin Exp Res 2001, 25: 1 423-30

17. DiPadova C, Worner TM, Julkunen RJ, Lieber CS: Effects of fasting and chronic alcohol consumption on the first-pass metabolism of ethanol. Gastroenterology 1987, 92:1 I 69-73

18. Chumlea WC, Guo SS, Zeller CM, Reo NV, Baumgartner RN, Garry PJ, Wang J, Pierson RN Jr, Heymsfield SB, Siervogel RM: Total body water reference values and prediction equations for adults. Kidney Int 200I, 59:2250-8

19. Norberg A: Cllinical Pharmcokinetics of Intravenous Ethanol. Stockholm: Karolinska University Press; 2001

20. Bosron WF, Crabb DW, Li TK: Relationship between kinetics of liver alcohol dehydrogenase and alcohol metabolism. Pharmacol Biochem Behav 1983, I 8:223-7

21. Chinard FP, Thaw CN, Delea AC, Perl W: Intrarenal volumes of distribution and relative diffusion coefficients of monohydric alcohols. Circ Res 1969, 25:343-57

22. Sasaya S, Yagi H, Yamaguchi M, Kigawa G, Nakano H, Midorikawa T, Nagasaki $H$, Kumada $K$ : Liver function assessed by increased rate of portal venous blood flow after oral intake of glucose. Eur J Surg 2000, 166:1 12-8
23. Pugliese D, Ohnishi K, Tsunoda T, Sabba C, Albano O: Portal hemodynamics after meal in normal subjects and in patients with chronic liver disease studied by echo-Doppler flowmeter. Am J Gastroenterol 1987, 82: 1052-6

24. Gaiani S, Bolondi L, Li Bassi S, Santi V, Zironi G, Barbara L: Effect of meal on portal hemodynamics in healthy humans and in patients with chronic liver disease. Hepatology 1989, 9:815-9

25. Jones AW, Jonsson KA: Food-induced lowering of blood-ethanol profiles and increased rate of elimination immediately after a meal. J Forensic Sci 1994, 39:1084-93

26. Hahn RG, Norberg A, Gabrielsson J, Danielsson A, Jones AW: Eating a meal increases the clearance of ethanol given by intravenous infusion. Alcohol Alcohol 1994, 29:673-7

27. Jonsson F, Johanson G: Physiologically Based Modeling of the Inhalation Kinetics of Styrene in Humans Using a Bayesian Population Approach. Toxicol Appl Pharmacol 2002, I 79:35-49

28. Sheiner LB, Ludden TM: Population pharmacokinetics/dynamics. Annu Rev Pharmacol Toxicol 1992, 32:185-209

29. Jones AW, Hahn RG, Stalberg HP: Distribution of ethanol and water between plasma and whole blood; inter- and intra-individual variations after administration of ethanol by intravenous infusion. Scand J Clin Lab Invest 1990, 50:775-80

30. Wagner JG: Lack of first-pass metabolism of ethanol at blood concentrations in the social drinking range. Life $\mathrm{Sci}$ 1986, 39:407-14

31. Levitt MD, Li R, DeMaster EG, Elson M, Furne J, Levitt DG: Use of measurements of ethanol absorption from stomach and intestine to assess human ethanol metabolism. Am J Physiol I997, 273:G95I-7

\section{Pre-publication history}

The pre-publication history for this paper can be accessed here:

http://www.biomedcentral.com/1472-6904/2/4/prepub
Publish with BioMed Central and every scientist can read your work free of charge

"BioMedcentral will be the most significant development for disseminating the results of biomedical research in our lifetime." Paul Nurse, Director-General, Imperial Cancer Research Fund

Publish with BMC and your research papers will be: - available free of charge to the entire biomedical community

- peer reviewed and published immediately upon acceptance

- cited in PubMed and archived on PubMed Central

- yours - you keep the copyright

Submit your manuscript here:

http://www.biomedcentral.com/manuscript/
BioMedcentral.com editorial@biomedcentral.com 